\title{
Sudden Death in a Patient with Undiagnosed Diabetes, Acute Pancreatitis and Acute Adrenalitis
}

\author{
Cynthia Jin ${ }^{1}$, Long Jin ${ }^{2 *}$ \\ ${ }^{1}$ Tulane University, New Orleans, USA \\ ${ }^{2}$ Department of Pathology, Louisiana State University Health Sciences Center-Shreveport, Shreveport, LA, USA \\ Email: *ljin@lsuhsc.edu
}

How to cite this paper: Jin, C. and Jin, L. (2019) Sudden Death in a Patient with Undiagnosed Diabetes, Acute Pancreatitis and Acute Adrenalitis. Forensic Medicine and Anatomy Research, 7, 63-67. https://doi.org/10.4236/fmar.2019.74010

Received: July 15, 2019

Accepted: August 11, 2019

Published: August 14, 2019

Copyright () 2019 by author(s) and Scientific Research Publishing Inc. This work is licensed under the Creative Commons Attribution International License (CC BY 4.0).

http://creativecommons.org/licenses/by/4.0/

\section{c) (i) Open Access}

\begin{abstract}
It has been well documented that acute pancreatitis and diabetic ketoacidosis (DKA) co-occur, and both of them present either as a cause or result of the other [1] [2]. Recently, hypertriglyceridemia has been reported to co-occur as well [3] [4]; the involvement of acute adrenalitis in the setting of acute pancreatitis and DKA has not been reported. We report a case of sudden death due to diabetic ketoacidosis, acute pancreatitis and acute adrenalitis in a 37-year-old female. She was found unresponsive and breathless in bed by her mother at their residence. At the time of the autopsy, both lungs were expanded and sigmoid diverticulosis was discovered on gross examination. Histology of the pancreas and the adrenal glands revealed acute inflammation despite appearing normal upon gross examination. The postmortem toxicology report showed high levels of acetone $(12 \mathrm{mg} / \mathrm{dL})$ in the postmortem blood and extremely high levels of glucose $(>500 \mathrm{mg} / \mathrm{dL})$ in the postmortem vitreous fluid. This is the first reported case of fatal diabetic ketoacidosis co-occurring with acute pancreatitis and acute adrenalitis.
\end{abstract}

\section{Keywords}

Diabetic Ketoacidosis (DKA), Acute Pancreatitis, Acute Adrenalitis, Autopsy

\section{Introduction}

Since halfway through the twentieth century, it has been reported that diabetic ketoacidosis co-occurs with acute pancreatitis [1] [2] [5] [6] [7] [8]. Meta-analyses have found that patients with pre-existing diabetes mellitus have a $94 \%$ increased risk of suffering acute pancreatitis than patients without diabetes mellitus [9]. More specifically, there was an $84 \%$ increased risk of suffering acute 
pancreatitis in patients with type 2 diabetes [10]. Recently, hypertriglyceridemia has been reported to co-occur as well, making the matter more complicated and forming the comorbidity triad of diabetic ketoacidosis, hypertriglyceridemia and acute pancreatitis [5] [6]. A few case reports indicate that severe acute pancreatitis triggers the development of severe diabetic ketoacidosis because some of the patients in the case reports did not have pre-existing diabetes [2] [7]. It is still unclear whether acute pancreatitis is the cause or the result of uncontrolled diabetes present as diabetic ketoacidosis. We report a fatal diabetic ketoacidosis with acute pancreatitis and acute adrenalitis in a 37-year old female. To date, this is the first case of diabetic ketoacidosis co-occurring with acute pancreatitis and acute adrenalitis that has been reported in the English literature.

\section{Case Report}

The patient was a 37-year-old African American female with a clinical history of hypertension. She was found in bed, unresponsive and breathless by her mother at their residence. She had complained of abdominal pain and taken an anti-acid tablet before going to bed the previous night. An autopsy was performed at the Pathology Department of LSUHSC-Shreveport upon the request of the local Coroner's office. Postmortem gross examination was largely unremarkable, with the only findings being mildly expanded lungs and sigmoid diverticulosis. Her heart and kidneys reveal no significant histopathologic changes, despite the clinical history of hypertension. On microscopic examination, hematoxylin and eo$\sin (\mathrm{H} \& \mathrm{E})$ sections of the pancreas revealed diffuse acute inflammatory infiltrate with neutrophils in the acini, ducts and some islets (Figure 1 and Figure 2). Sections of the adrenal glands also revealed acute inflammatory infiltrate with neutrophils, mainly in the medulla and zone of reticularis (Figure 3 and Figure 4). Special stains of Gram, Acid Fast Bacilli (AFB) Fite and Grocott's Methenamine Silver (GMS) did not reveal any organisms. Toxicology revealed high level

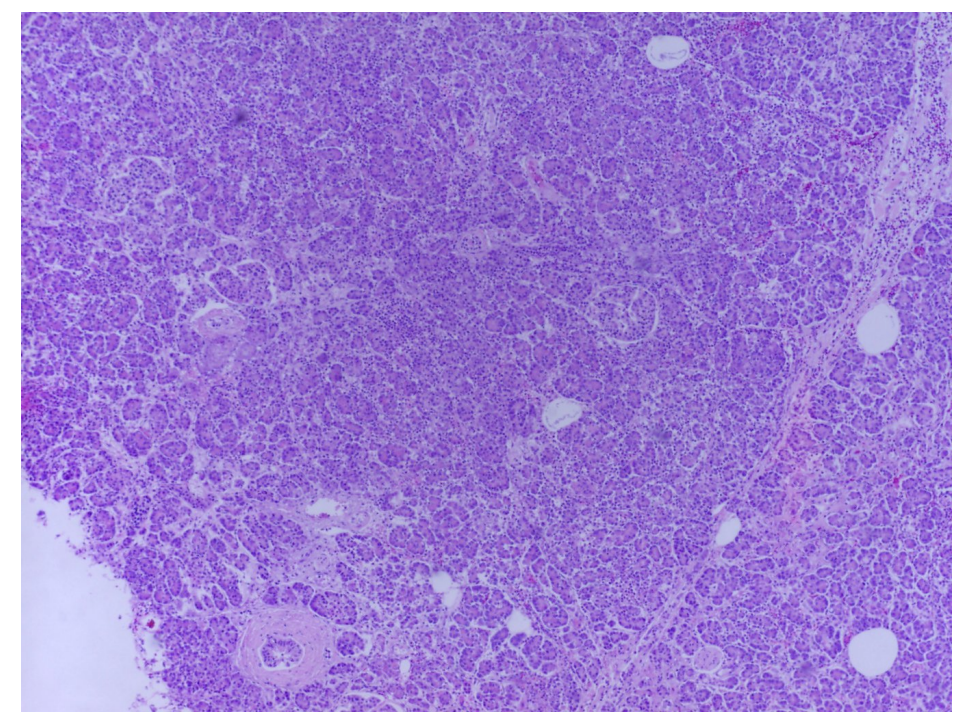

Figure 1. Acute inflammatory infiltrate with neutrophils in pancreas $(\mathrm{H} \& \mathrm{E}, \times 4)$. 


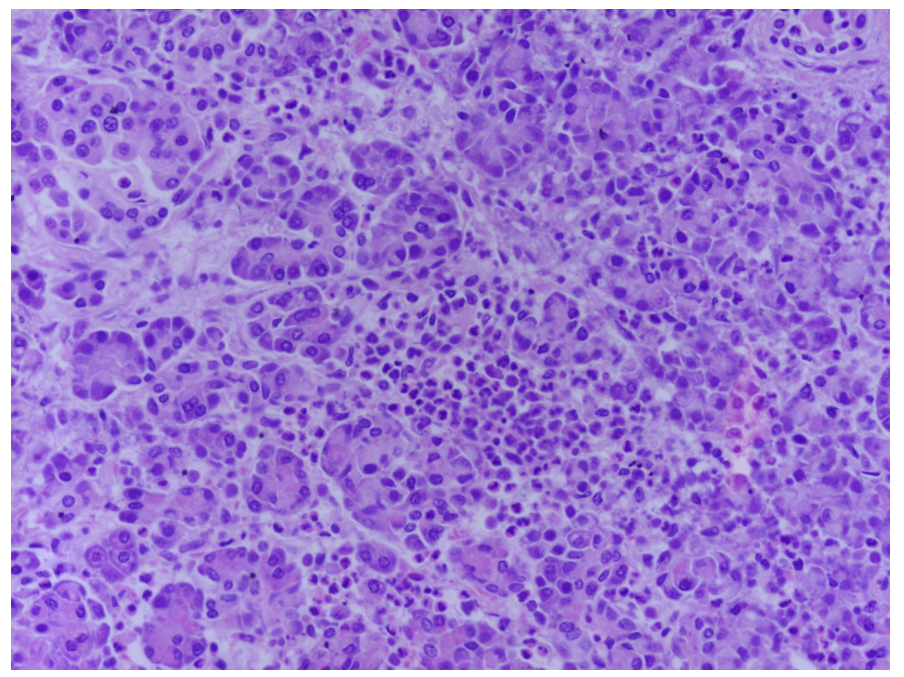

Figure 2. Acute inflammatory infiltrate with neutrophils in pancreas (H \& E, $\times 20)$.

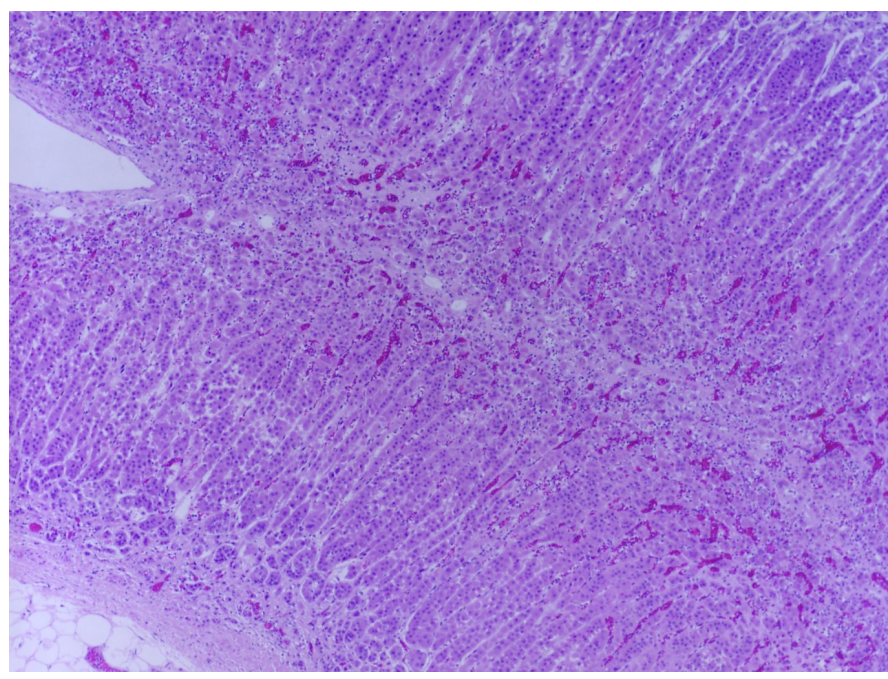

Figure 3. Acute inflammatory infiltrate with neutrophils in adrenal gland $(\mathrm{H} \& \mathrm{E}, \times 4)$.

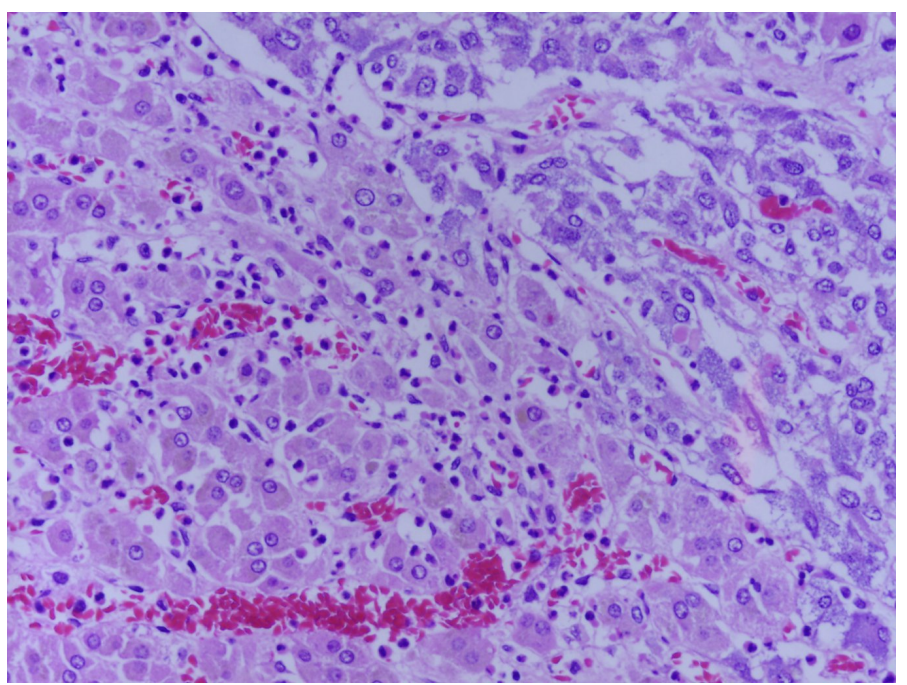

Figure 4. Acute inflammation infiltrate with neutrophils in adrenal gland ( $\mathrm{H} \& \mathrm{E}, \times 20)$. 
of acetone $(12 \mathrm{mg} / \mathrm{dL})$ in the postmortem blood and extremely high levels of glucose $(>500 \mathrm{mg} / \mathrm{dL})$ in the postmortem vitreous fluid, consistent with diabetic ketoacidosis. The cause of death was determined to be diabetic ketoacidosis. Acute pancreatitis and acute adrenalitis are significant contributory factors. The manner of death is natural.

\section{Discussion}

Since the initial clinical symptom of acute pancreatitis and diabetic ketoacidosis could be nonspecific and present solely as abdominal pain, the diagnosis of either acute pancreatitis or diabetic ketoacidosis by a clinician is very difficult and challenging, especially in the previously undiagnosed diabetic patient.

Our patient was an undiagnosed diabetic patient. The high levels of acetone in postmortem blood raised the suspicion of diabetic ketoacidosis and prompted us to check the vitreous glucose level, and examine the histology of the pancreas. The pancreas appeared normal on gross examination. The histology of the pancreas revealed acute inflammatory infiltrate with neutrophils in the acini and islets. There was no hemorrhage or necrosis. Bug stain panel with Gram, AFB Fite and GMS was negative.

Adrenal dysfunction could be fatal due to the adrenal gland's vital role of producing hormones to regulate metabolism, responding to stress and controlling blood pressure, etc. Adrenal insufficiency is mostly secondary to autoimmune adrenalitis [11]. Acute adrenal infection is rare, though it can be caused by a variety of pathogens, including bacteria, viruses, fungi and parasites [11] [12]. Among them, the classic Waterhouse-Friderichsen syndrome presents as acute bilateral adrenal hemorrhages caused by $N$. meningitidis. The disease can be caused by other bacteria, such as group A Streptococci and Pneumococci, etc [13] [14]. In our patient, both adrenal glands appeared normal on gross examination. The histology of both adrenal glands revealed acute inflammatory infiltrate with neutrophils in the medulla and cortex, mainly in reticularis. There was no hemorrhage or necrosis. Bug stain panel with Gram, AFB Fite and GMS was negative. The complete absence of hemorrhage and necrosis in this patient indicated that her acute pancreatitis and acute adrenalitis were in their early stages.

Unfortunately, blood culture was not performed during the procedure, largely due to lack of clinical indication. The cause of acute pancreatitis and acute adrenalitis is still unknown. Our speculation is that the patient was in an early stage of sepsis. It has yet to be reported that the acute adrenalitis is associated with diabetes ketoacidosis and acute pancreatitis. Further research to understand the pathophysiology in this unique triad of diabetic ketoacidosis, acute pancreatitis and acute adrenalitis will be pursued, despite its rare occurrence.

\section{Conclusion}

This is the first reported case of the unique triad of diabetic ketoacidosis, acute pancreatitis, and acute adrenalitis. This case further highlights the importance of histology examinations of the important organs, even if there are no significant 
findings on gross examination.

\section{Conflicts of Interest}

The authors declare no conflicts of interest regarding the publication of this paper.

\section{References}

[1] Tully, G.T. and Lowenthal, J.J. (1958) The Diabetic Coma of Acute Pancreatitis. Annals of Internal Medicine, 48, 310-319. https://doi.org/10.7326/0003-4819-48-2-310

[2] Hughes, P.D. (1961) Diabetic Acidosis with Acute Pancreatitis. British Journal of Surgery, 49, 90-91. https://doi.org/10.1002/bjs.18004921320

[3] Butler, D.C., Emanuel, A.J., Self, S.E. and Batalis, N.I. (2017) The Interplay between Diabetes and Pancreatitis: Two Case Reports of Sudden, Natural Deaths and a Review of the Literature. Journal of Forensic Sciences, 62, 519-524. https://doi.org/10.1111/1556-4029.13268

[4] Simons-Linares, C.R., Jang, S., Sanaka, M., Bhatt, A., Lopez, R., Vargo, J., Stevens, T. and Chahal, P. (2019) The Triad of Diabetes Ketoacidosis, Hypertriglyceridemia and Acute Pancreatitis. How Does It Affect Mortality and Morbidity? A 10-Year Analysis of the National Impatient Sample. Medicine, 98, e14378. https://doi.org/10.1097/MD.0000000000014378

[5] Davidson, A.I. (1964) Diabetic Coma without Ketoacidosis in a Patient with Acute Pancreatitis. British Medical Journal, 1, 356. https://doi.org/10.1136/bmj.1.5379.356

[6] Maclean, D., Murinson, J. and Griffiths, P.D. (1973) Acute Pancreatitis and Diabetic Ketoacidosis in Accidental Hypothermia, and Hypothermic Myxoedema. British Medical Journal, 4, 757-761. https://doi.org/10.1136/bmj.4.5895.757

[7] Nielson, O.S. and Simonsen, E. (1969) A Case of Transient Diabetes Mellitus in Connection with Acute Pancreatitis. Acta Medica Scandinavica, 185, 459-462. https://doi.org/10.1111/j.0954-6820.1969.tb07365.x

[8] Nair, S., Yadav, D. and Pitchumoni, C.S. (2000) Association of Diabetic Ketoacidosis and Acute Pancreatitis: Observations in 100 Consecutive Episodes of DKA. Journal of Gastroenterology, 95, 2795-2800. https://doi.org/10.1111/j.1572-0241.2000.03188.x

[9] Xue, L., Sheng, Y., Dai, H., Cao, H., Liu, Z. and Li, Z. (2012) Risk of Development of Acute Pancreatitis with Preexisting Diabetes: A Meta-Analysis. European Journal of Gastroenterology \& Hepatology, 24, 1092-1098. https://doi.org/10.1097/MEG.0b013e328355a487

[10] Yang, L., He, Z., Tang, X. and Liu, J. (2013) Type 2 Diabetes Mellitus and the Risk of Acute Pancreatitis: A Meta-Analysis. European Journal of Gastroenterology \& Hepatology, 25, 225-231. https://doi.org/10.1097/MEG.0b013e32835af154

[11] Arlt, W. and Allolio, B. (2013) Adrenal Insufficiency. The Lancet, 361, 1881-1893. https://doi.org/10.1016/S0140-6736(03)13492-7

[12] Paolo, W.F. and Nosanchuk, J.D. (2006) Adrenal Infections. International Journal for Infectious Disease, 10, 343-353. https://doi.org/10.1016/j.ijid.2005.08.001

[13] Friderichsen, C. (1955) Waterhouse-Friderichsen Syndrome. Acta Endocrinologica, 18, 489-492. https://doi.org/10.1530/acta.0.0180482

[14] Doherty, S. (2001) Fatal Pneumococcal Waterhouse-Friderichsen Syndrome. Emergency Medicine, 13, 237-239. https://doi.org/10.1046/j.1442-2026.2001.00219.x 